\title{
Phonon dispersion of a two-dimensional boron sheet on $\mathrm{Ag}(111)$
}

\author{
Takashi Aizawa $\odot^{*}$ and Shigeru Suehara $\odot^{\dagger}$ \\ International Center for Materials Nanoarchitectonics, National Institute for Materials Science, 1-1 Namiki, \\ Tsukuba, Ibaraki 305-0044, Japan \\ Shigeki Otani \\ Research Center for Functional Materials, National Institute for Materials Science, 1-1 Namiki, \\ Tsukuba, Ibaraki 305-0044, Japan
}

(Received 30 April 2021; accepted 4 June 2021; published 15 June 2021)

\begin{abstract}
Two-dimensional (2D) atomically thin boron layers designated as borophene or boraphene, attract great interest as promising post-graphene $2 \mathrm{D}$ materials. The borophene layer phonon structure has not yet been clarified by experimentation in spite of its importance for application for possible superconduction and thermal technologies. For this work we measured, by experimentation, the phonon dispersion of an incommensurate three-domain $\chi_{3}$-borophene formed on $\mathrm{Ag}(111)$ using high-resolution electron energy loss spectroscopy. Measured phonons were analyzed using first-principles theoretical calculations. Because the phonon band of the substrate $\mathrm{Ag}(111)$ lies in a low-energy region, the borophene phonon is well separated from the substrate. For our calculations, a commensurate slab supercell model was adopted, the periodicity of which was $\operatorname{Ag}(111) 3 \times 3$. The lattice parameter of the model was determined using experimentally obtained phonon data. The highest frequency phonon of an isolated borophene layer was fitted to the data as a function of the electronic charge in it. The best fitted lattice parameter was used in the supercell model. The model well reproduced the experimentally observed phonon dispersion. No marked anomaly was found in the phonon structure.
\end{abstract}

DOI: 10.1103/PhysRevMaterials.5.064004

\section{INTRODUCTION}

Since the beginning of the 21 st century, two-dimensional (2D) materials of one or several atomic thickness have become a main stream both in fundamental and application research fields. Following the amazing success of graphene, atomically thin elemental materials designated as "Xenes" have been explored extensively in anticipation of various applications: silicene $(\mathrm{Si})$, germanene $(\mathrm{Ge})$, stanene $(\mathrm{Sn})$, phosphorene $(\mathrm{P})$, borophene or boraphene (B), and so on [1]. Among these Xenes, borophene is most interesting because of its characteristic properties [2-6] and many promising applications for gas sensors, hydrogen storage in fuel cells, superconductors, electrodes in alkaline metal batteries, catalysts for hydrogen evolution or oxygen reduction $[7,8]$, and biomedical devices [9].

In 2001, considerably high critical temperature $\left(T_{c}\right)$ of superconductivity was reported for magnesium diboride $\left(\mathrm{MgB}_{2}\right)$ [10]. Subsequently, several theoretical works explained its mechanisms [11-13]. They clarified that high

\footnotetext{
*aizawa.takashi@nims.go.jp

†suehara.shigeru@nims.go.jp

Published by the American Physical Society under the terms of the Creative Commons Attribution 4.0 International license. Further distribution of this work must maintain attribution to the author $(s)$ and the published article's title, journal citation, and DOI.
}

frequency B-B stretching vibration mode in the honeycomb boron layer in $\mathrm{MgB}_{2}$ interacts strongly with its electronic $\sigma$ band, responding to high $T_{c}$ superconductivity. Actually, B is the lightest atom among the Xenes, resulting in high-frequency phonons also in borophene. Theoretical calculations predicted that superconductivity can be retained in a Mg-intercalated bilayer borophene [14] or borophene on $\mathrm{Ag}(111)$ [15]. The electron-phonon interaction in borophene is predicted to be larger than in $\mathrm{MgB}_{2}$ [16].

Existence of the spontaneously formed monoatomic boron layer (boron layer termination) was found in 1998-2001 for some group-5 (Ta) [17,18] ( $\mathrm{Nb})$ [19] and group-6 (W) [20] transition-metal diboride $\left(\mathrm{TMB}_{2}\right)(0001)$ singlecrystal surfaces. However, the surface is terminated by the transition-metal layer on the group-4 $\mathrm{TMB}_{2}(0001)$ [19,21]. We have produced a borophene (boraphene) layer on the metal-terminated group- $4 \mathrm{TMB}_{2}, \mathrm{ZrB}_{2}(0001)$ by depositing atomic boron, which exhibited a $\sqrt{3} \times \sqrt{3}\left(R \pm 30^{\circ}\right)$ commensurate periodicity with the substrate [22]. Phonon dispersions of such borophenes on $\mathrm{TMB}_{2}(0001)$ have been measured using high-resolution electron energy loss spectroscopy (HREELS) [19,22]. They have been analyzed using first-principles density functional theory (DFT) and density functional perturbation theory (DFPT) calculations in 2010 [23]. Agreement between the calculated and measured phonon dispersion was excellent. The clarified borophene structure on $\mathrm{ZrB}_{2}(0001)$ is a $\mathrm{B}_{7}$ layer: one adatom in the $\sqrt{3} \times \sqrt{3}$ unit cell of the honeycomb boron layer. That is the same as the $\alpha$ sheet with one additional vacancy in the unit 
cell, corresponding to the vacancy concentration of $2 / 9$ in the recent nomenclature for borophene allotropes [2,24].

Independently from the reports presented above, borophene was studied theoretically in the early 2010s [24-27]. It has been synthesized through experimentation on a more popular substrate, $\operatorname{Ag}(111)$, since 2015 [28,29]. The borophene layer is fabricated by depositing atomic B on the $\operatorname{Ag}(111)$ single crystal substrate, similarly to our earlier method of boraphene synthesis on $\mathrm{ZrB}_{2}(0001)$ [22]. The only experimentally obtained data for the borophene phonon on $\operatorname{Ag}(111)$ were those measured using Raman spectroscopy, as reported very recently [30]. Because the momentum of photon is small in Raman or infrared spectroscopy, they can assess only around the $\Gamma$ point. However, the theoretical calculations predicted that the large electron-phonon interaction does not necessarily occur at the $\bar{\Gamma}$ point, especially in nonhoneycomb borophenes [14,15,31]. Additionally, to ascertain whether the $\bar{\Gamma}$-point phonon shows large electron-phonon interaction, or not, the dispersion curves must be addressed apart from the $\bar{\Gamma}$ point. Therefore, measurement of full phonon dispersion is necessary to elucidate the borophene properties. Nevertheless, it has never been reported for borophene on $\operatorname{Ag}(111)$.

This report presents the full phonon dispersion relations of $\chi_{3}$-borophene formed on $\operatorname{Ag}(111)$. We follow Ref. [25] for the borophene allotrope notation. The phonon dispersion was measured using HREELS, and was calculated using DFT and DFPT. The calculation can well reproduce the experimentally obtained data under a supercell structure model in which the lattice parameter is adjusted.

\section{METHOD}

The experiment was conducted using the same apparatus as that used in our earlier work $[19,22,32-34]$. Briefly described, an HREELS spectrometer (Delta 0.5; SPECS GmbH) was held in a magnetically shielded ultrahigh vacuum (UHV) chamber with base pressure of less than $4 \times 10^{-9} \mathrm{~Pa}$. The energy resolution was about $2 \mathrm{meV}$ in the full-width at half-maximum (FWHM) of the elastic (zero-loss) peak. In the HREELS chamber, a microchannel-plate backview lowenergy electron diffraction (LEED) apparatus was used to align the azimuthal orientation of the sample. The sample was prepared in another UHV chamber, the base pressure of which was $2-4 \times 10^{-8} \mathrm{~Pa}$. The preparation chamber was connected to the HREELS chamber through a transfer tube. The following can be performed in the preparation chamber: reflection high-energy electron diffraction (RHEED), Auger electron spectroscopy (AES), $\mathrm{Kr}^{+}$ion bombardment, $\mathrm{Ag}$ deposition from a Knudsen cell (K-cell, EF 40C1; PREVAC) with a pyrolytic boron nitride (PBN) crucible, and B deposition from an electron-heated evaporator (EFM 3; Scienta Omicron, Inc.). The sample can be heated to $2500 \mathrm{~K}$ in the preparation chamber by electron bombardment of the rear. The sample temperature was measured using an optical pyrometer without calibration.

A $\mathrm{NbC}_{0.95}$ (hereafter $\mathrm{NbC}$ ) (111) single-crystal surface was used as a substrate for Ag film epitaxy. The $\mathrm{NbC}$ single crystal was grown using an rf-heated floating zone method [35]. A disk $(\phi 8-9 \mathrm{~mm} \times \mathrm{t} 1-1.5 \mathrm{~mm})$ having $(111) \pm 1^{\circ}$ faces was cut from the grown rod after orienting the $\langle 111\rangle$ direction by x-ray back-Laue method. The disk face was polished with diamond paste to a mirror finish. After ultrasonic degreasing with acetone, the $\mathrm{NbC}(111)$ substrate was cleaned in UHV by repeated heating at about $1900 \mathrm{~K}$. Ag (99.99\%; Sigma-Aldrich Corp.) was deposited using the K-cell on the clean $\mathrm{NbC}(111)$ substrate. B was deposited on the Ag epitaxial film from a $\beta$-boron crystal rod. After the sample was characterized with RHEED and AES, it was transferred to the HREELS in the UHV to measure the phonon spectra.

First-principles calculations were conducted using QUANTUM-ESPRESSO ver. 6.5 suite [36], with a generalized gradient approximation, the Perdew-Burke-Ernzerhof (PBE) functional for the exchange correlation [37], and the PBE ultrasoft pseudopotentials [38]. After checking convergence, cutoff energies for wave function ( $\left.E_{\mathrm{CUT}}\right)$ and charge density $\left(E_{\text {CUT }}^{\rho}\right)$ were taken, respectively, at 35 and 320 Ry. The sampling $\boldsymbol{k}$ points were $18 \times 18 \times 18$ for the bulk $\mathrm{Ag}$, and $6 \times 6 \times 1$ for the $3 \times 3 \times n$ supercell calculations. Permissible residual force in the structure optimization was $10^{-5} \mathrm{Ry} / \mathrm{a}$.u. In the phonon dispersion calculations, the sampling $\boldsymbol{q}$ points were $3 \times 3 \times 1$ for the supercell model. The calculations were performed on a large cluster computer in our institute. For visualization the structure models, the VESTA 3 package was used [39]. For the calculated atomic structure, the electron population analysis was performed using the LOBSTER package [40].

\section{RESULTS AND DISCUSSION}

\section{A. Experiment results}

After heating in UHV, the $\mathrm{NbC}(111)$ exhibited a clear $1 \times 1$ RHEED pattern. No contamination was detected using AES. It has been established that the clean $\mathrm{NbC}(111)$ is terminated with the metal atom $(\mathrm{Nb})$ layer [41]. Even though the lattice parameter of $\operatorname{Ag}(111)\left(a_{\mathrm{Ag}(111)}=288.4 \mathrm{pm}\right.$ [42]) is smaller $(-8.7 \%)$ than that of $\mathrm{NbC}(111)\left(a_{\mathrm{NbC}(111)}=315.9\right.$ pm [35]), deposition of $\mathrm{Ag}$ at room temperature caused in the epitaxial growth for which the relationship was $(111)_{\mathrm{Ag}} \|$ $(111)_{\mathrm{NbC}}$ and $[1 \overline{1} 0]_{\mathrm{Ag}} \|[\overline{1} 10]_{\mathrm{NbC}}$. This reverse stacking is probably created by the $\mathrm{NbC}$ structure: When the $\mathrm{Nb}$ sublattice stacking is $\mathrm{ABC} . .$. , the carbon is located in the alternate site as $\mathrm{AcBaCb} . .$. , where the small letter denotes the carbon layer. The Ag stacking is probably affected by the carbon layer below the surface. The deposition for $30 \mathrm{~min}$ at the Ag cell temperature of $900^{\circ} \mathrm{C}$ yielded an epitaxial $\operatorname{Ag}(111)$ film of about $100 \mathrm{~nm}$ thick. Figure 1(a) exhibits the RHEED pattern and the AES spectrum (lower panel) of the epitaxial $\operatorname{Ag}(111)$ film, indicating a clean $\operatorname{Ag}(111)$ surface. The in-plane lattice constant $a$ of the grown film was estimated at 285-288 nm from the RHEED patterns, identical to that of $\operatorname{Ag}(111)$ within the experimental error. This suggests that the grown $\operatorname{Ag}(111)$ is almost free from the stress of the lattice mismatch in this film thickness.

Boron was deposited on the clean $\operatorname{Ag}(111)$ epitaxial film surface at $620 \mathrm{~K}$. After 10-20 min of the deposition, the RHEED pattern changed to an incommensurate pattern close to $3 \times 3$ [Figs. 1(b) and 1(c)]. A B $K V V$ Auger signal was apparently observed in the AES. The LEED pattern of this borophene [Fig. 1(d)] resembles that reported in the 


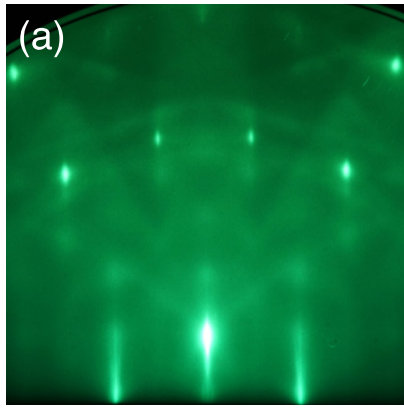

$\mathrm{Ag}$
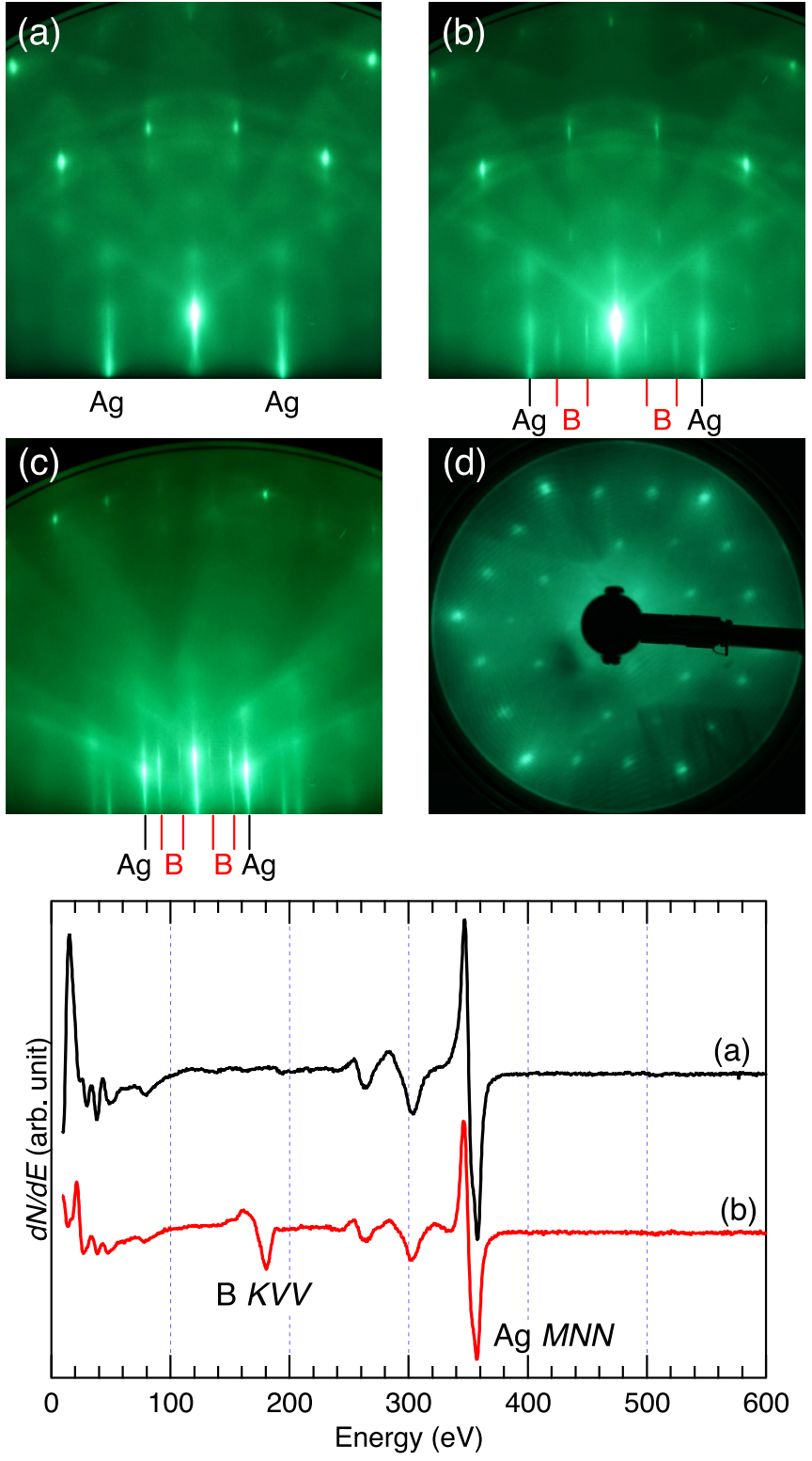

FIG. 1. RHEED (a)-(c), LEED (d), and AES (lower panel) of (a) clean $\mathrm{Ag}(111)$ surface and (b)-(d) borophene covered $\mathrm{Ag}(111)$. Primary electron energies $\left(E_{0}\right)$ are $15 \mathrm{keV}$ for RHEED and $113.7 \mathrm{eV}$ for LEED. The azimuthal orientations are [121] for (a) and (b), and [101] for (c). The $\operatorname{Ag}(111)$ fundamental diffractions and those from borophene are represented, respectively, by black and red lines as a guide to the eyes. The LEED pattern has some pincushion distortion because of the planar screen.

literature for $\chi_{3}$-borophene on $\operatorname{Ag}(111)$ [43], but it differs greatly from that for $\beta_{12}$-borophene [44]. The LEED pattern seems to be $3 \times 3$ at first glance. However, it is not exactly $3 \times 3$, but incommensurate because the diffraction streaks from borophene [red marks in Fig. 1(c)] are not distributed uniformly between the $\mathrm{Ag}$ fundamentals. The specular HREEL spectrum is shown in Fig. 2. Three small loss peaks were observed at $23.1 \mathrm{meV}\left(187 \mathrm{~cm}^{-1}\right), 62.5 \mathrm{meV}$ $\left(504 \mathrm{~cm}^{-1}\right)$, and $88.0 \mathrm{meV}\left(709 \mathrm{~cm}^{-1}\right)$. These frequencies correspond respectively to the $B_{g}^{2}, B_{g}^{1}$ (or $A_{g}^{4}$ ), and $A_{g}^{3}$ modes observed in $\chi_{3}$-borophene on $\operatorname{Ag}(111)$ using Raman scattering

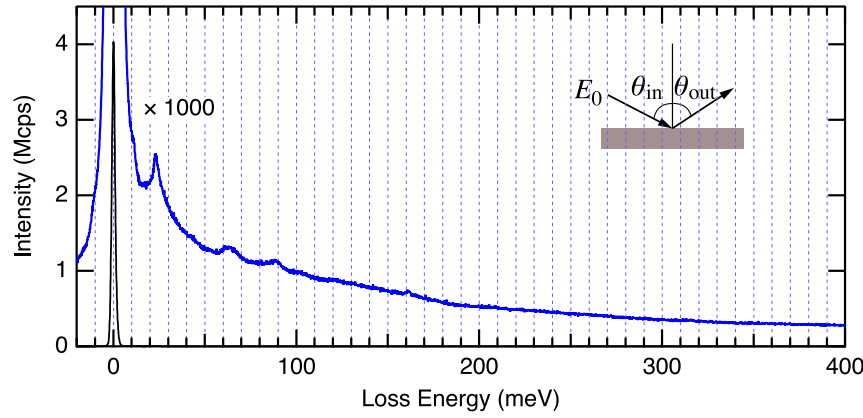

FIG. 2. Specular HREEL spectrum of the borophene on $\operatorname{Ag}(111) . E_{0}=10.0 \mathrm{eV}$ and $\theta_{\text {in }}=\theta_{\text {out }}=75^{\circ}$.

spectroscopy [30]. From these results we inferred our sample as $\chi_{3}$-borophene. The small trace at $160.8 \mathrm{meV}$ (1297 $\mathrm{cm}^{-1}$ ) in the HREEL spectrum can be assigned to a B-O stretching vibration from a very small amount of oxygen residue.

Figure 3 exhibits a typical series of off-specular HREELS data taken at the primary electron energy $\left(E_{0}\right)$ of $10 \mathrm{eV}$. Several $E_{0} \mathrm{~s}(6,10$, and $17 \mathrm{eV})$ were applied to measure as many phonon modes as possible. In Fig. 4 the loss peak energies are shown versus momentum transfer parallel to the surface, which corresponds to the phonon wave vector, representing the experimentally measured phonon dispersion relations [45]. Because of the large mass of $\mathrm{Ag}$, the substrate $\mathrm{Ag}(111)$ phonon band is located below $22 \mathrm{meV}$ [46]. Therefore, almost all observed phonon modes are attributable to overlayer borophene. The lowest lying mode below $10 \mathrm{meV}$ corresponds well to the surface acoustic mode $\left(S_{1}\right.$ : Rayleigh mode) of $\mathrm{Ag}(111)$ observed in inelastic $\mathrm{He}$ atom scattering [47] and HREELS [48,49] experiments. Some borophene phonon modes have large energy dispersion, suggesting considerably strong B-B bonds in the borophene layer. This strong B-B bonding is also reflected in the high-frequency phonon up to $150 \mathrm{meV}\left(1210 \mathrm{~cm}^{-1}\right)$ around the $\bar{\Gamma}$ point, which also agrees well with the $A_{g}^{1}$ phonon frequency of the $\chi_{3}$-borophene on $\mathrm{Ag}(111)$ measured using Raman spectroscopy [30].

In the case of $\chi_{3}$-borophene on $\operatorname{Ag}(111)$, the borophene orientation is examined using scanning tunneling microscopy (STM) [29]: the row of vacancy site is aligned along $\operatorname{Ag}\langle 11 \overline{2}\rangle$. Because $\operatorname{Ag}(111)$ has threefold symmetry, three equivalent domains invariably exist, for which the vacancy row is aligned with each of three $\langle 11 \overline{2}\rangle$ directions [43]. Therefore, one of three domains of the $\chi_{3}$-borophene aligns as $(\Gamma-X)_{\mathrm{B}} \|[1 \overline{2} 1]_{\mathrm{Ag}}$ and $(\Gamma-Y)_{\mathrm{B}} \|[10 \overline{1}]_{\mathrm{Ag}}$, whereas the other two domains are deviated $\pm 120^{\circ}$ from the highly symmetric axes. This three-domain structure makes the phonon structure very complex because of the different overlapping domains.

\section{B. Calculation results}

\section{Modeling}

As the near $3 \times 3$ LEED pattern [Fig. 1(d)] shows, the $3 \times 3$ periodicity of the substrate $\operatorname{Ag}(111)$ is expected to coincide roughly with the incommensurate borophene layer. 


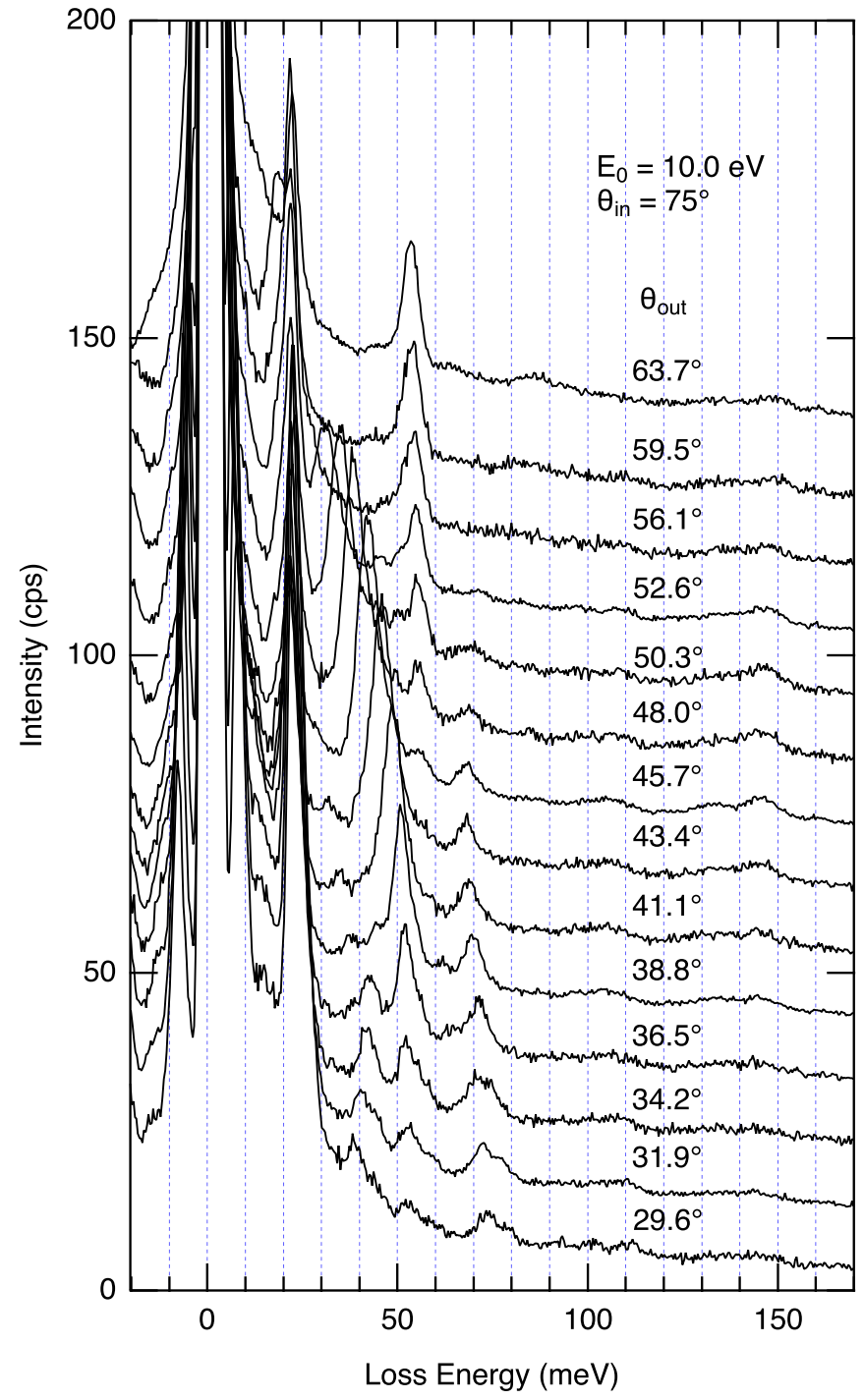

FIG. 3. Typical series of off-specular HREEL spectra of the borophene on $\operatorname{Ag}(111)$ along the $\langle 11 \overline{2}\rangle(\bar{\Gamma}-\bar{M})$ azimuth of the substrate.

This finding might be readily apparent from the proposed model [29]. Therefore, we took the commensurate structural model as an approximation, where $3 \times 3$ of $\operatorname{Ag}(111)$ coincides with $(2 \boldsymbol{c}+3 \boldsymbol{d},-3 \boldsymbol{c}-2 \boldsymbol{d})$ of $\chi_{3}$-borophene, as presented in Fig. 5(a). The unit cell includes $20 \mathrm{~B}$ atoms and $9 n \mathrm{Ag}$ atoms in $n$ substrate layers. In that case, because the substrate thickness is far larger than the overlayer, the overlayer lattice constant is forced to fit with the substrate if the whole system is optimized. This situation is unsuitable for investigation of the overlayer properties. In this work, the substrate lattice constant parallel to the surface was forcibly fit to the overlayer one, which was calculated in an isolated borophene.

The incommensurate structure itself suggests that the direct bond between B and Ag should be weak. Therefore, the charge transfer from the substrate was first considered. After introducing the electronic charge into a free-standing $\chi_{3}$-borophene, its cell parameters and atomic positions were optimized; then the phonon frequency was calculated.

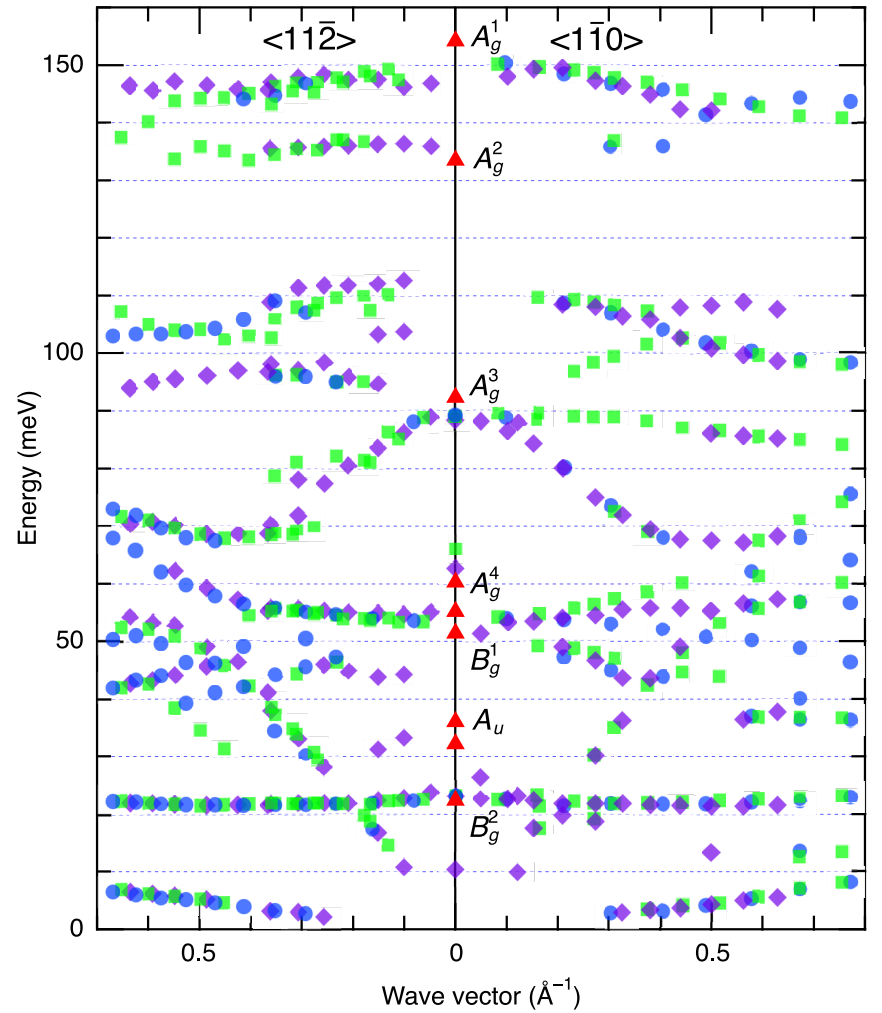

FIG. 4. Experimentally measured phonon dispersion of borophene on $\mathrm{Ag}(111) . E_{0}=6.0 \mathrm{eV}$ (purple diamonds), $10.0 \mathrm{eV}$ (green squares), and $17.0 \mathrm{eV}$ (blue circles). Red triangles represent the Raman spectroscopy data [30].

The lattice optimization was performed under the restriction of $|\boldsymbol{a}|=|\boldsymbol{b}|$. The angle between $\boldsymbol{a}$ and $\boldsymbol{b}$ was fixed at $120^{\circ}$. It was put into the $3 \times 3$ commensurate supercell later.

We devoted particular attention to the highest frequency phonon as the criterion, the experimentally obtained value of which is about $150 \mathrm{meV}\left(1210 \mathrm{~cm}^{-1}\right)$. This phonon corresponds to the B-B stretching vibration in the borophene, which is sensitive to the B-B distance. Figure 6 presents the lattice constant and the highest frequency phonon energy at $\bar{\Gamma}$ point versus the excess electron charge in the free-standing $\chi_{3}$-borophene sheet. From this result we infer the phonon energy best fit to the experiment at the excess electron charge of $5 e^{-}$in the $\mathrm{B}_{20}$ borophene: $1 / 4 e^{-}$per one boron atom. At this charge state, the lattice parameter is $|\boldsymbol{a}|=|\boldsymbol{b}|=0.850$ $\mathrm{nm}$. In calculations hereinafter, the cell parameter is fixed at this value, which is $3.4 \%$ smaller than the calculated bulk Ag lattice $(a=414.92 \mathrm{pm})$. At this cell parameter, the $\mathrm{Ag}$ substrate is under some stress, so that the calculated phonon for the Ag has less meaning.

As the next step, the borophene layer was put on a fivelayer $\operatorname{Ag}(111)$ slab to produce a supercell. Between the slabs, a vacuum layer of more than $1.6 \mathrm{~nm}$ was maintained. The bottom three layers $(\mathrm{Ag} 3-5)$ of the $\mathrm{Ag}$ slab were fixed to save calculation time. The interlayer distance of these layers is fixed to the calculated bulk value. The overlayer borophene (B) and the top two Ag (Ag1-2) layers were moved freely to minimize the total energy. Even after the structural optimization, the borophene overlayer remained in an almost 


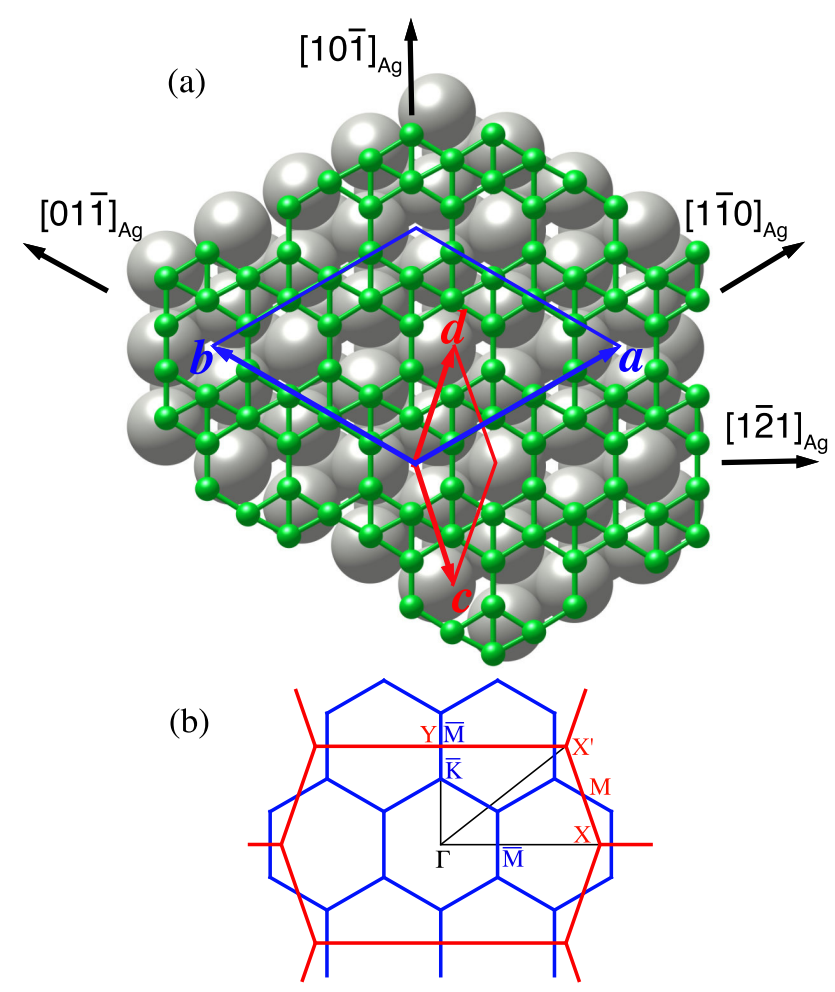

FIG. 5. (a) Plan-view supercell structure model and (b) surface Brillouin zones. For the blue rhombus, vectors $\boldsymbol{a}$ and $\boldsymbol{b}$ represent the unit cell in the calculation. A red one (vectors $\boldsymbol{c}$ and $\boldsymbol{d}$ ) is the unit cell of $\chi_{3}$-borophene overlayer. Gray and green balls, respectively, represent $\mathrm{Ag}$ and $\mathrm{B}$ atoms.

flat shape. The final structure is shown in Fig. 5. The mean interlayer distances of the optimized structure are presented in Table I. Corrugation in the borophene layer is slight: the almost flat $\chi_{3}$-borophene on $\operatorname{Ag}(111)$ is predicted by this calculation.

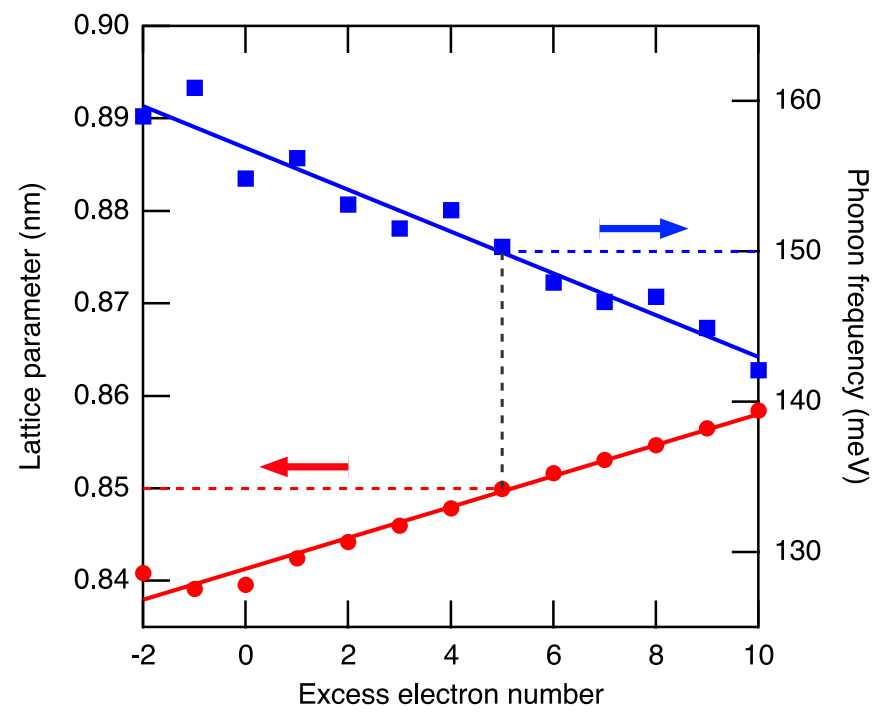

FIG. 6. Calculated lattice constant (red circles) and frequency of the highest energy at $\bar{\Gamma}$ point (blue squares) of the free-standing $\mathrm{B}_{20} \chi_{3}$-borophene with respect to the excess electron charge.
TABLE I. Calculated mean interlayer distances $(d)$ and rumpling $(r)$ in the layer of the optimized slab model.

\begin{tabular}{|c|c|c|c|c|c|c|c|c|}
\hline Layer & B & & Ag1 & & $\mathrm{Ag} 2$ & & Ag3 & \\
\hline$d(\mathrm{pm})$ & & 242.3 & & 244.1 & & 245.7 & & 239.5 \\
\hline$r(\mathrm{pm})$ & 11.4 & & 9.6 & & 4.2 & & 0 & \\
\hline
\end{tabular}

\section{Phonon dispersion}

The calculated phonon dispersion relations are presented in Fig. 7. Because of the three-domain structure of $\chi_{3}$-borophene on $\mathrm{Ag}(111)$, two independent domains exist along the high symmetry axes. In Fig. 7 (a) the $\bar{\Gamma}-\bar{M}$ (left-hand side) axis aligns in the vacancy row direction. The $\bar{\Gamma}-\bar{K}-\bar{M}$ (right-hand side) axis is perpendicular to the vacancy row. The imaginary phonon modes appear because the bottom three layers of the substrate Ag are fixed. The dense band located below $20 \mathrm{meV}$ is assigned to $\mathrm{Ag}$ vibration, which is meaningless here.

The $\chi_{3}$-borophene has a striped structure: Along the vacancy row are strongly bonded boron belts, whereas the belts are connected with fewer bonds in the vacancy row region. The phonon dispersion of Fig. 7(a) exhibits this feature clearly. Along the vacancy row (left side), the phonon modes disperse considerably, while along the perpendicular direction (right side), the dispersions are flat, suggesting phonon insulation by the vacancy rows. In the other domains [Fig. 7(b)], the dispersion is large irrespective of the direction, because the axes are deviated from the vacancy row. In the real sample, these domains coexist, so that these dispersions must be overlapped with the weight of 1:2. As a result, the calculated dispersion becomes overly complicated. We further analyzed the calculated phonon dispersion by projection into each kind of atom and vibrational direction.

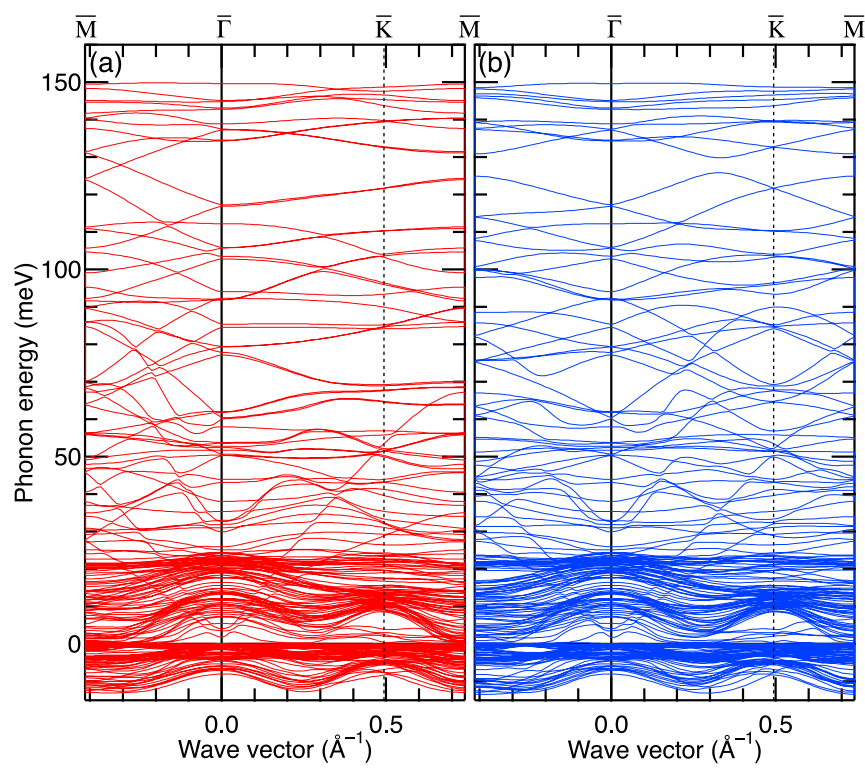

FIG. 7. Calculated phonon dispersion curves of (a) domain 1 (along and perpendicular to the vacancy row) and (b) domains 2 and $3\left( \pm 120^{\circ}\right.$ from the domain 1$)$. 

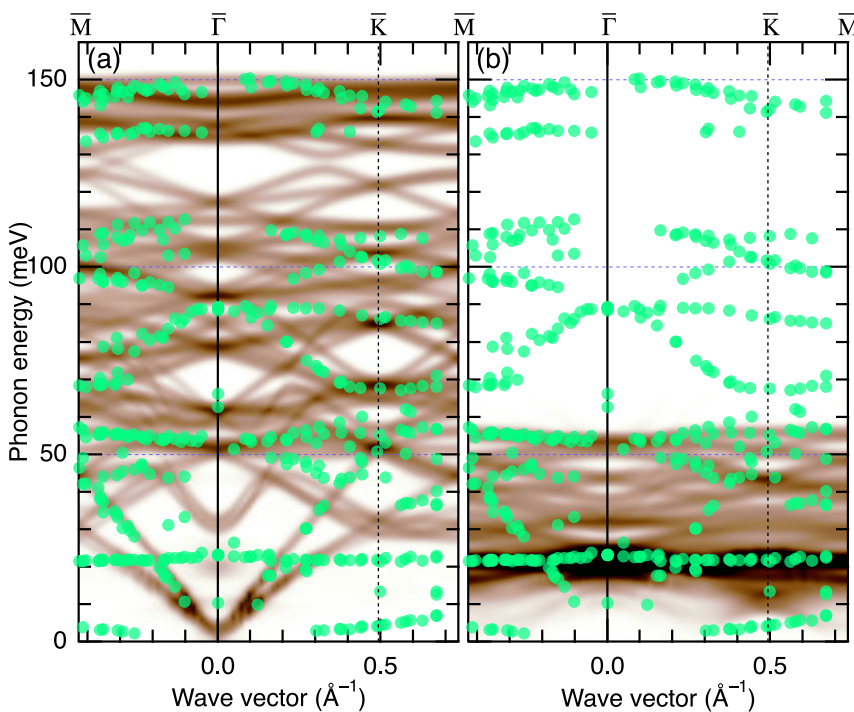

FIG. 8. Calculated partial-density phonon dispersion (brown curves) of the borophene layer and the experimentally obtained data (green dots): (a) in-plane modes and (b) out-of-plane modes. Color darkness represents the phonon density.

The phonon densities of state (DOS) were calculated with square amplitudes of each atom's vibrational eigenvector. The DOS were summed up for the layers and components of interest. The phonon dispersion is weighted with the DOS, resulting in the projected phonon dispersion of the subject layer. This projected phonon is then broadened with Gaussian of $2 \mathrm{meV}$ FWHM to simulate the resolution used for experimentation. Figure 8 exhibits the resultant partial DOS phonon dispersion of (a) the borophene in-plane vibrations and (b) the borophene out-of-plane vibrations. Whereas the in-plane phonon spreads in the wide energy range from 0 to $150 \mathrm{meV}$, the out-of-plane modes are restricted in the energy range from 10 to $55 \mathrm{meV}$. Although not all the calculated phonon modes are detected by the HREELS experiment, almost all dark brown modes (high DOS modes) have the corresponding data points, indicating that the reproducibility of the calculation is very good.

For the in-plane phonon dispersion [Fig. 8(a)], the longitudinal acoustic (LA) mode rising from the zero energy at $\bar{\Gamma}$ is especially well reproduced. From the experimentally obtained slope of this mode, the sound velocity of the LA mode is estimated at $16.7 \mathrm{~km} / \mathrm{s}$. This value is considerably large but less than that of graphene $(19.9 \mathrm{~km} / \mathrm{s})$ [50]. In the out-of-plane phonon [Fig. 8(b)], the dispersionless mode around $20 \mathrm{meV}$ is assigned to the stretching vibration of $\mathrm{B}-\mathrm{Ag}$, which is located energetically at the top edge of the $\operatorname{Ag}(111)$ substrate phonon band, and which is therefore resonant with it. Because of the resonance, the energy of this mode is broadened in the calculation. The difference of charge between B and Ag makes this mode slightly dipole active. It was therefore observed clearly in the specular HREELS as shown in Fig. 2.

\section{Electronic structure}

The calculated electronic band structure of the isolated $\chi_{3}{ }^{-}$ borophene is portrayed in Fig. 9. The result is almost identical

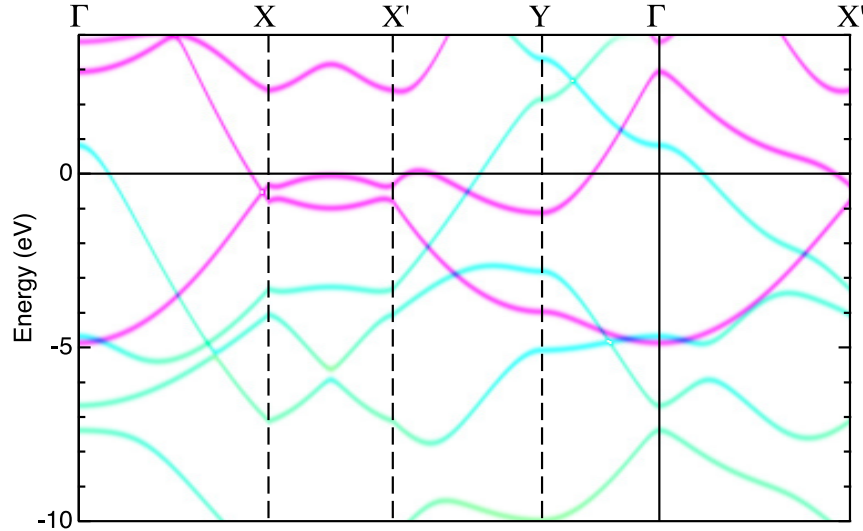

FIG. 9. Calculated electronic band structure of an isolated $\chi_{3}-$ borophene. The projected density is represented with the color darkness of magenta (B $2 p_{\mathrm{z}}$ ), cyan (B $2 p_{\mathrm{xy}}$ ), and yellow (B $\left.2 s\right)$. The energy scale is taken as $E_{f}$ (Fermi energy) $=0 \mathrm{eV}$.

to what has been reported earlier $[31,43]$. The magenta $\pi$ band $\left(p_{z}\right)$ is distinguished completely from the $\sigma$ band ( $p_{x y}$ and $s)$. A part of the cyan $p_{x y}$ band becomes greenish, indicating mixture with the yellow $s$ band, which can be interpreted as

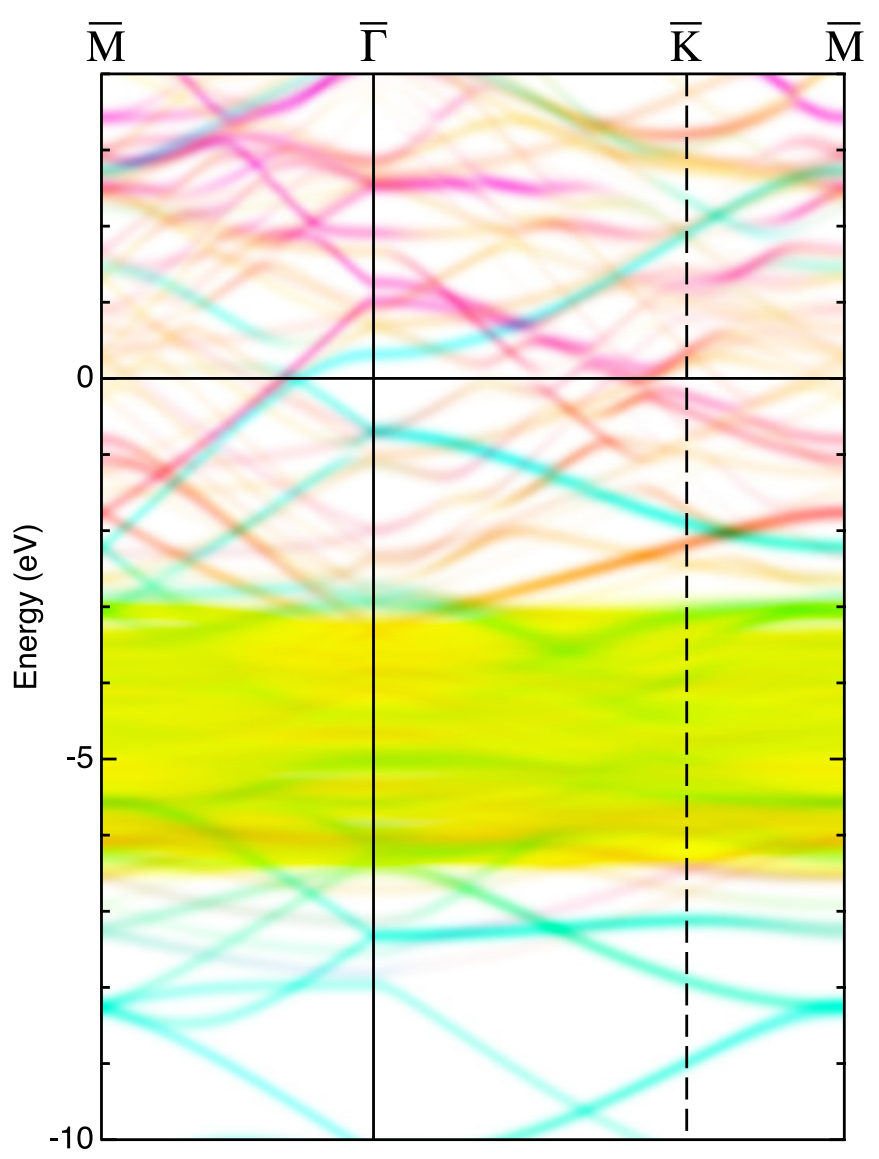

FIG. 10. Projected electronic band structure of the $\operatorname{Ag}(111) 3 \times$ 3- $\chi_{3} \mathrm{~B}$ slab for the domain 1 [Fig. 7(a)]. The color depth represents the projected density of the $\mathrm{B} 2 p_{\mathrm{z}}$ (magenta), B $2 p_{\mathrm{xy}}$ (cyan), and the subsurface layer $\mathrm{Ag} s+p+d$ (yellow). The energy scale is taken as $E_{f}=0 \mathrm{eV}$. 
the $s p^{2}$ hybrid orbital. The electronic band presents characteristic features of a planar 2D material such as graphene. Figure 10 portrays the calculated projection band structure of the slab model $\left(\mathrm{B}_{20}\right.$ borophene layer +5 layers $3 \times 3 \mathrm{Ag}$ substrate). The dense yellow band between -3 and $-6.5 \mathrm{eV}$ is the $\mathrm{Ag} d$ band. In other energy regions, the borophene electronic bands are well resolved. The cyan B $p_{x y}$ band seems to be independent from the substrate: no greenish band is apparent. However, the magenta $\mathrm{B} p_{z}$ band partly changes its color to orange or red, indicating that this band mixes with the substrate (yellow) band. Although the borophene $\pi$ band interacts more with the substrate than the $\sigma$ band does, the interaction itself seems weak because the $\pi$ band feature of the borophene is preserved to a considerable degree. The $\bar{\Gamma}-\bar{K}-\bar{M}$ path in Fig. 10 corresponds to the $\Gamma-Y$ path in Fig. 9. For example, the $\pi$ band running from $\sim+1 \mathrm{eV}$ at $\bar{\Gamma}$ to $\sim-1 \mathrm{eV}$ at $\bar{M}$ (Fig. 10) corresponds well to the $\pi$ band of the isolated borophene from $\sim+3 \mathrm{eV}$ at $\Gamma$ to $\sim-1 \mathrm{eV}$ at $Y$ in Fig. 9.

The Löwdin charge analysis of this model indicated the boron charge of about 0.1 electrons per atom. This value is smaller than that expected in the isolated borophene layer calculation to determine the lattice constant (Fig. 6; 0.25 electrons per atom). Probably the real charge transfer between the borophene layer and the substrate is not so large. Additionally, the overlap integrals between B and Ag are small. These calculation results suggest that the interaction between the borophene overlayer and the substrate $\mathrm{Ag}$ is so weak that the intrinsic features of the borophene are expected to remain to a considerable degree.

\section{SUMMARY}

To summarize, the $\chi_{3}$-borophene layer was fabricated on a $\operatorname{Ag}(111)$ epitaxial film surface. The phonon dispersion relations of this $\chi_{3}$-borophene were measured experimentally using HREELS. The $3 \times 3 \times 6$ (one $\mathrm{B}$ and five Ag layers) slab supercell model was constructed. Its phonon dispersion was calculated using first-principles DFT and DFPT. Because of the three-domain structure of the $\chi_{3} \mathrm{~B} / \mathrm{Ag}(111)$, the phonon dispersion was remarkably complex. However, the projected overlayer borophene modes fit fairly well to the experimentally obtained data. In neither the experiment nor the calculation was any clear anomaly found in the phonon dispersion.

The calculated electronic structure indicated that the borophene $\pi$ band interacts more with the substrate Ag than the borophene $\sigma$ band. However, the interaction seems weak; moreover, the $\pi$ band feature of the borophene layer is preserved to a considerable degree. The ionicity of the borophene and the overlap population between $\mathrm{B}$ and $\mathrm{Ag}$ is also small.

\section{ACKNOWLEDGMENTS}

T.A. acknowledges the financial support by Japan Science and Technology Agency Mirai Program Grant No. JPMJMI19A. The calculations were performed on the $\mathrm{Nu}$ merical Materials Simulator at National Institute for Materials Science.
[1] N. R. Glavin, R. Rao, V. Varshney, E. Bianco, A. Apte, A. Roy, E. Ringe, and P. M. Ajayan, Adv. Mater. 32, 1904302 (2020).

[2] Z. Zhang, E. S. Penev, and B. I. Yakobson, Chem. Soc. Rev. 46, 6746 (2017).

[3] Z. Xie, X. Meng, X. Li, W. Liang, W. Huang, K. Chen, J. Chen, C. Xing, M. Qiu, B. Zhang, G. Nie, N. Xie, X. Yan, and H. Zhang, Research 2020, 2624617 (2020).

[4] I. Matsuda and K. Wu (eds.), 2D Boron: Boraphene, Borophene, Boronene (Springer International, Cham, 2021).

[5] X.-F. Zhou, X. Dong, A. R. Oganov, Q. Zhu, Y. Tian, and H.-T. Wang, Phys. Rev. Lett. 112, 085502 (2014).

[6] X.-F. Zhou, A. R. Oganov, Z. Wang, I. A. Popov, A. I. Boldyrev, and H.-T. Wang, Phys. Rev. B 93, 085406 (2016).

[7] Z.-Q. Wang, T.-Y. Lü, H.-Q. Wang, Y. P. Feng, and J.-C. Zheng, Front. Phys. 14, 33403 (2019).

[8] P. Ranjan, J. M. Lee, P. Kumar, and A. Vinu, Adv. Mater. 32, 2000531 (2020).

[9] M. Tatullo, B. Zavan, F. Genovese, B. Codispoti, I. Makeeva, S. Rengo, L. Fortunato, and G. Spagnuolo, Appl. Sci. 9, 3446 (2019).

[10] J. Nagamatsu, N. Nakagawa, T. Muranaka, Y. Zenitani, and J. Akimitsu, Nature (London) 410, 63 (2001).

[11] J. M. An and W. E. Pickett, Phys. Rev. Lett. 86, 4366 (2001).

[12] T. Yildirim and O. Gülseren, J. Phys. Chem. Solids 63, 2201 (2002).

[13] T. Soda and Y. Fukumoto, Prog. Theor. Phys. 111, 707 (2004).
[14] J.-H. Liao, Y.-C. Zhao, Y.-J. Zhao, H. Xu, and X.-B. Yang, Phys. Chem. Chem. Phys. 19, 29237 (2017).

[15] Y. Zhao, S. Zeng, and J. Ni, Appl. Phys. Lett. 108, 242601 (2016).

[16] M. Gao, Q.-z. Li, X.-w. Yan, and J. Wang, Phys. Rev. B 95, 024505 (2017).

[17] H. Kawanowa, R. Souda, S. Otani, and Y. Gotoh, Phys. Rev. Lett. 81, 2264 (1998).

[18] H. Kawanowa, R. Souda, K. Yamamoto, S. Otani, and Y. Gotoh, Phys. Rev. B 60, 2855 (1999).

[19] T. Aizawa, W. Hayami, and S. Otani, Phys. Rev. B 65, 024303 (2001).

[20] H. Kawanowa, Y. Gotoh, S. Otani, and R. Souda, Surf. Sci. 433435, 661 (1999).

[21] W. Hayami, R. Souda, T. Aizawa, and T. Tanaka, Surf. Sci. 415, 433 (1998).

[22] T. Aizawa, S. Suehara, S. Hishita, and S. Otani, J. Phys. Condens. Matter 20, 265006 (2008).

[23] S. Suehara, T. Aizawa, and T. Sasaki, Phys. Rev. B 81, 085423 (2010).

[24] E. S. Penev, S. Bhowmick, A. Sadrzadeh, and B. I. Yakobson, Nano Lett. 12, 2441 (2012).

[25] X. Wu, J. Dai, Y. Zhao, Z. Zhuo, J. Yang, and X. C. Zeng, ACS Nano 6, 7443 (2012).

[26] Y. Liu, E. S. Penev, and B. I. Yakobson, Angew. Chem. Int. Ed. 52, 3156 (2013). 
[27] Z. Zhang, Y. Yang, G. Gao, and B. I. Yakobson, Angew. Chem. Int. Ed. 54, 13022 (2015).

[28] A. J. Mannix, X.-F. Zhou, B. Kiraly, J. D. Wood, D. Alducin, B. D. Myers, X. Liu, B. L. Fisher, U. Santiago, J. R. Guest, M. J. Yacaman, A. Ponce, A. R. Oganov, M. C. Hersam, and N. P. Guisinger, Science 350, 1513 (2015).

[29] B. Feng, J. Zhang, Q. Zhong, W. Li, S. Li, H. Li, P. Cheng, S. Meng, L. Chen, and K. Wu, Nat. Chem. 8, 563 (2016).

[30] S. Sheng, J.-B. Wu, X. Cong, Q. Zhong, W. Li, W. Hu, J. Gou, P. Cheng, P.-H. Tan, L. Chen, and K. Wu, ACS Nano 13, 4133 (2019).

[31] E. S. Penev, A. Kutana, and B. I. Yakobson, Nano Lett. 16, 2522 (2016).

[32] T. Aizawa, S. Suehara, and S. Otani, J. Phys. Chem. C 118, 23049 (2014).

[33] T. Aizawa, S. Suehara, and S. Otani, J. Phys. Condens. Matter 27, 305002 (2015).

[34] T. Aizawa, S. Suehara, and S. Otani, Phys. Rev. Materials 3, 014005 (2019).

[35] S. Otani, T. Tanaka, and Y. Ishizawa, J. Cryst. Growth 62, 211 (1983).

[36] P. Giannozzi, O. Baseggio, P. Bonfà, D. Brunato, R. Car, I. Carnimeo, C. Cavazzoni, S. de Gironcoli, P. Delugas, F. Ferrari Ruffino, A. Ferretti, N. Marzari, I. Timrov, A. Urru, and S. Baroni, J. Chem. Phys. 152, 154105 (2020).

[37] J. P. Perdew, K. Burke, and M. Ernzerhof, Phys. Rev. Lett. 77, 3865 (1996).
[38] K. F. Garrity, J. W. Bennett, K. M. Rabe, and D. Vanderbilt, Comput. Mater. Sci. 81, 446 (2014).

[39] K. Momma and F. Izumi, J. Appl. Crystallogr. 44, 1272 (2011).

[40] S. Maintz, V. L. Deringer, A. L. Tchougréeff, and R. Dronskowski, J. Comput. Chem. 37, 1030 (2016).

[41] W. Hayami, R. Souda, T. Aizawa, S. Otani, and Y. Ishizawa, Phys. Rev. B 47, 13752 (1993).

[42] W. P. Davey, Phys. Rev. 25, 753 (1925).

[43] B. Feng, J. Zhang, S. Ito, M. Arita, C. Cheng, L. Chen, K. Wu, F. Komori, O. Sugino, K. Miyamoto, T. Okuda, S. Meng, and I. Matsuda, Adv. Mater. 30, 1704025 (2018).

[44] B. Feng, J. Zhang, R.-Y. Liu, T. Iimori, C. Lian, H. Li, L. Chen, K. Wu, S. Meng, F. Komori, and I. Matsuda, Phys. Rev. B 94, 041408(R) (2016).

[45] H. Ibach and D. L. Mills, Electron Energy Loss Spectroscopy and Surface Vibrations (Academic, New York, 1982), p. 257.

[46] J. Xie, S. de Gironcoli, S. Baroni, and M. Scheffler, Phys. Rev. B 59, 970 (1999).

[47] R. B. Doak, U. Harten, and J. P. Toennies, Phys. Rev. Lett. 51, 578 (1983).

[48] L. Chen, L. L. Kesmodel, and J.-S. S. Kim, Surf. Sci. 350, 215 (1996).

[49] M. W. G. Ponjée, C. F. J. Flipse, A. W. D. van der Gon, and H. H. Brongersma, Phys. Rev. B 67, 174301 (2003).

[50] X. Cong, Q.-Q. Li, X. Zhang, M.-L. Lin, J.-B. Wu, X.-L. Liu, P. Venezuela, and P.-H. Tan, Carbon 149, 19 (2019). 